\title{
Development of nitride-sensors for monitoring in control systems
}

\author{
Monzer Krishan', Ahmad Alkhawaldeh², Abdel-Hamid Soliman ${ }^{3}$ \\ ${ }^{1}$ Department of Mechatronics Engineering, Al-Balqa Applied University, Amman, Jordan \\ ${ }^{2}$ Department of Chemistry, Jerash University, Jerash, Jordan \\ ${ }^{3}$ Department of Mechatronics Engineering, Staffordshire University, Stoke-on-Trent, United Kingdom \\ ${ }^{1}$ Corresponding author \\ E-mail: ${ }^{1}$ drkrishan@bau.edu.jo, ${ }^{2}$ Ahmad.alkawalda@yahoo.com, ${ }^{3}$ a.soliman@staffs.ac.uk
}

Received 20 March 2020; received in revised form 10 August 2020; accepted 18 August 2020

DOI https://doi.org/10.21595/jme.2020.21384

Check for updates

Copyright $(\mathbb{C} 2020$ Monzer Krishan, et al. This is an open access article distributed under the Creative Commons Attribution License, which permits unrestricted use, distribution, and reproduction in any medium, provided the original work is properly cited.

\begin{abstract}
Sensors become integrated through the control condition arrangement, either for visual, mechanical, biological, or chemical applications. New stuff is designed for detection, such as Diluted Magnetic Semiconductors (DMS), which are considered attractive candidates that consist of traditional 111- V, II-VI, or group IV semiconductors. Manganese Mn-doped GaN (Mn.Gac.N) epitaxial velum has unique magnetic, visual and chemical properties for the control of systems intelligently in detector design. The subject area of the magnetic properties of MnxGal-xN is on a large scale available; there are only a few studies on the visual properties and electrochemical properties of MnxGal-xN epitaxial velums. Where $\mathrm{MnGaN}$ velums were used in spintronic and opto-electronic applications according to their magnetic characterization and constructed $\mathrm{MnGaN}$ electrodes have drop-fabric potentials for potentiometric sensor applications, since they have good performance as ion-selective electrodes. The electrical and magnetic properties that allow the control of electron spin as well as compliant period, makes the materials ideal for spintronic applications. Designing such spintronic and optoelectronic devices based on MnxGal-xN requires a broader agreement of physical, visual, electrical and chemical properties of epitaxial velums that are still seldom found in the literature. This bailiwick displays the potential use of $\mathrm{MnGaN}$ semiconductor as an all solid-state potentiometric sensor for measuring anions in solutions in the control-engineering field.
\end{abstract}

Keywords: diluted magnetic semiconductors (DMS), manganese Mn-doped GaN (Mn.Gac.N), spintronic, opto-electronic devices control system, measurement.

\section{Introduction}

Metal-Organic Chemical Vapor Deposition (MOCVD) epitaxial film MnGaN has been examined by Secondary Electron Emission, Ultraviolet (UV) measurements, X-Ray Diffraction (XRD), Open Circuit Potential (OCP), and Energy Dispersive X-ray Spectrometer (EDS), for its crystalline quality and surface structure, optical characterization, and electrochemical properties [1].

The perfect crystal structure, magnetic properties, and distribution of elements are clearly discussed and demonstrated by high-resolution XRD and EDS measurements, which also provide the practical data for the relative calculation. Moreover, optical characterizations, for example, the energy band gap of $\mathrm{MnGaN}$ changes in the range $1 . \mathrm{geV}-3.5 \mathrm{eV}$, are revealed by the UV measurements [2]. It is a great discovery for semiconductor nanotechnology of traditional III - V, II-VI, or group IV semiconductors [3].

The electrochemical properties of MnxGal_xN thin films are for the first time presented by Ion Selective Electrodes (ISE) [5]. This study demonstrates the potential utilization of MnGaN semiconductor as an all solid-state potentiometric device for measurement of anions in solutions in the control-engineering field.

The semiconductor is a solid material that has electrical conductivity in the middle of a conductor and an insulator. Semiconductor devices over a large scale range either permanently or 
dynamically [6]. Semiconductor units are tremendously important in engineering science. Examples range from computers to cellular earpieces to digital audio role players. Semiconductor devices are primary in modem electrical senses of devices [7, 8]. The mix of various semiconductor types together creates gadgets with special electrical properties, which allow restraint of electrical signals. Silicon is used to create most commercial semiconductor units but loads of other materials are used as well [9].

The Diluted magnetic semiconductors (DMS) are considered as an important candidate for the next generation of electronic devices which consist of the traditional Trine - V, II-VI, or group IV semiconductors [6]. Among these DMS materials, manganese Mn-doped GaN (MnxGal_xN) epitaxial films have gained growing interests. It is one of the ideal semiconductor materials which are under intense investigation in the Diluted Magnetic Semiconductors (DMS) inquiry [10]. It allows the interplay of electronic and magnetic properties and is structurally compatible with most epitaxial full-grown III-V semiconductors [3, 4]. Thus, nanostructures of GaN-based DMS have gained attention from the expectation of new magnetic and magneto -visual properties, due to the combining of the quantum parturiency and the magnetic properties peculiar to this material.

Several years ago, the field of electronics twirl (Spintronics) aimed to use spin of carriers for electronic gimmick, which has emerged as the new frontier in device physics for the future integrated technology. The thought of utilizing the spin of carrier's spintronic devices has led to efforts in fabricating and investigating appropriate new semiconductor materials [11]. To make Mn xGal-xN a potentially ideal material for spin-injection applications, such as spin p-n diodes, spin transistors, spin detectors, spin Light Emitting Diodes (LEDs), more suggested applications include: semiconductor-based spin valves, spin field effect transistors, and even spin qubits, to be used as the basic building blocks for quantum computing which utilize both information processing and data storage within one material system [12]. A combination of conventional semiconductors with magnetic materials would be very desirable. The research of analyzing the Mn storey-transition free energy by optical measurements in the ultraviolet-visible region reported the strongly localized twist $(S=5 / 2)$ that the Mn 3cf electrons could couple with free carriers, resulting in an effective $\mathrm{Mn}-\mathrm{Mn}$ ferromagnetic interaction, and a duty period in the position of Fermi level with different Mn immersions [13]. According to the Linear Combination of Atomic Orbitals (LCAO) negatron band social structure of this semiconductor, the sum electron spin of the $\mathrm{Mn}$ corpuscle relies on the position of the fathometer level and inter-band transition, which are reported in [14].

Magnetic dimensions of $\mathrm{MnGaN}$ films are important for the optimization of the growing conditions of magnetic devices [15]. The property techniques used to measure the magnetic properties are the Vibrating Sample Magneto meter (VSM) and Superconducting Quantum Interference Device (SQUID). The VSM is versatile and can be used for electrical room temperature and visual control of ferromagnetism (FM) in the control organization. It requires an "Einstein" to manipulate data and produce a result [12]. The fast speed and huge database define high requirements of hardware of the computing system. Magnetism is already exploited in recording devices such as computer hard disks. Moreover, this information is recorded and stored as a tiny magnetized sphere of smoothing iron [15]. To access the knowledge, a browse mind detects the minute changes within the magnetic field because the disk twists underneath it. In addition, this evokes corresponding changes within the head's electrical resistance- an effect known as the magnetoresistance. Read heads, incorporating Giant Magneto Resistance (GMR) stuff, would be ready to sense a lot smaller magnetic line of business, allowing the storage capacity of a hard disk to extend from one to twenty GB [16]. GMR sensors have a wide range of applications: they have fast accurate position- and move- sensing of mechanical components in precision engineering and in robotics; all kinds of automotive sensors for fuel handling systems, antiskid systems, navigation, speed and electronic engine control; missile guidance in the current industry; position and motion sensing in computer telecasting games, as well as key-hole surgical process and post-intelligence agent-care applications. 


\section{Methodology}

To examine the performance of constructed MnxGal-xN electrodes via the different electron and chemical fields, a testing of sensitivity of inorganic and organic compounds around the range of electrolyte solutions (KF, $\mathrm{KNO}_{3}, \mathrm{KCI}, \mathrm{HOC}_{6} \mathrm{H}_{4} \mathrm{COONa}, \mathrm{KSCN}, \mathrm{CH}_{3} \mathrm{COOK}, \mathrm{KCIO}_{4}$, $\mathrm{KI}$, and $\mathrm{KBr}$ ) with concentrations of up $1 \times 10^{-1} \mathrm{M}$, up $1 \times 10^{-2} \mathrm{M}$, up $1 \times 10^{-3} \mathrm{M}$, up $1 \times 10^{-4} \mathrm{M}$, up $1 \times 10^{-5} \mathrm{M}$, and up $1 \times 10^{-6} \mathrm{M}$ ) was carried out.

\subsection{Electrochemical measurements (solutions)}

All electrolyte solutions were prepared by reagent-grade salts $\left(\mathrm{KF}, \mathrm{KNO}_{3}, \mathrm{KCI}\right.$, $\mathrm{HOC}_{6} \mathrm{H}_{4} \mathrm{COONa}, \mathrm{KSCN}, \mathrm{CH}_{3} \mathrm{COOK}, \mathrm{KCIO}_{4}, \mathrm{KBr}$, and $\mathrm{KI}$ ) with nanopure water (NPW, $18.2 \mathrm{MQ}$, Barnstead D119 11 Nanopure Diamond) in the laboratory. The procedure is using percentage by weight $(\mathrm{w} / \mathrm{v})$.

The formula was: [Mass of solute (g) / Volume of solution (mol)] x 100.

The highest initial concentration of solutions are made in $\mathrm{mol} / \mathrm{l}(\mathrm{M})$; then diluted to $10^{-2}-10^{-6}$ $\mathrm{M}$ for low constructions to complete calibration curves for each type of electrodes.

\subsection{Electrochemical cell assembly}

The three-electrode electrochemical cells, consisting of a reference electrode, a working electrode (semiconductor), and a counter electrode (also called an auxiliary electrode), were used for the electrochemical experiments. The potential measurements were done versus a Saturated Calomel Electrode (SCE) connected to the electrolyte by means of a salt bridge made of a glass capillary tube filled with a saturated KCI solution. The standard potential of the SCE reference electrode is $\mathrm{EO}(\mathrm{SCE})=0.242 \mathrm{~V}$ versus the Normal Hydrogen Electrode (NHE).

A Platinum wire electrode was used as the counter electrode. This electrode had a sufficiently large surface (more than 10 times larger than the surface of the working electrode) to ensure that its interface with the electrolyte did not influence the current-potential curves. To remove dissolved Oxygen from the solution and to keep the cell Oxygen-free throughout the experiments, the electrolytes were purged with Argon gas before the measurements, and Argon was blown over the solutions throughout the measurements. Before each series of measurements with a new electrolyte, the following procedures were carried out to ensure cleanliness of the equipment: 1) the Platinum counter electrode was thermally treated with a gas burner to remove species that could have been adsorbed on the electrode surface from the previous electrolyte, 2) the electrochemical cell was cleaned by submerging it into a hot bath $\left(90 \cdot{ }^{\circ} \mathrm{C}\right)$ of concentrated Hydrochloric and Sulphuric acids for one hour, and 3) the cell was rinsed with double distilled water and NPW in that sequence. All experiments were carried out at room temperature, which was almost constant at $21 \pm 1^{\circ} \mathrm{C}(16)$.

\subsection{Open circuit potential measurements}

The open circuit potential is the potential at which there is no current; that is, experiments based on the measurement of the electrical potential of an electrode against a reference electrode of the open circuit potential. It is a potentiometric experiment.

The measurements used 12542A Frequency Response Analyzer which provided the measurement of gain and phase and two points in the system. Electrochemical interface provided simultaneous voltage and current measurements.

The salt solutions $\left(\mathrm{KF}, \mathrm{KNO}_{3}, \mathrm{KCI}, \mathrm{HOC}_{6} \mathrm{f}_{4} \mathrm{COONa}, \mathrm{KSCN}, \mathrm{CH}_{3} \mathrm{COOK}, \mathrm{KCIO}_{4}, \mathrm{KI}\right.$, and $\mathrm{KBr})$ were prepared with deionized double-distilled water to produce concentrations between $\left(10^{-1}-10^{-6} \mathrm{M}\right)$. Electroanalytical chemical investigations were performed in a very typical threeelectrode cell that included Platinum counter electrode, a working electrode $(\mathrm{Mn} 0.1 \mathrm{Ga} 0.9 \mathrm{~N}$ or 
$\mathrm{Mn} 0.16 \mathrm{Ga} 0.84 \mathrm{~N})$, and a saturated calomel reference electrode. The potential measurements were done versus a Saturated Calomel Electrode (SCE) connected to the electrolyte by means of a salt bridge made of a glass capillary tube filled with a saturated KCL solution. The standard potential of the SCE reference electrode is $\mathrm{EO}(\mathrm{SCE})=0.242 \mathrm{~V}$ versus the Normal Hydrogen Electrode (NHE).

To remove dissolved Oxygen from the solutions and to keep the cell Oxygen-free throughout the experiments, Argon was blown over the solutions throughout the measurements, and therefore the electrolytes were purged with Argon before the experiments.

\subsection{Cyclic voltammetry (CV)}

Cyclic voltammetry is an electroanalytical method that utilizes nano/micro-electrodes in an unstirred solution so that the measured current and potential are limited by analyte diffusion at the electrode surface. The CV results of MnxGal-xN semiconductor can be directly related to the stability of the electrode in the studied test solutions. They were investigated by performing a series of range tests during which the potential of the electrode was scanned to highly negative and highly positive values (with respect to the electrode's OCP in the solution). They were also analyzed to determine the reactions, which contributed to the observed working electrode.

\section{Results and discussion}

Energy Dispersive Spectroscopy (EDS) is a chemical microanalysis technique utilized within conjunction for SEM to characterize the elemental composition of the analyzed sample. The EDS $\mathrm{X}$-ray detector measured the relative abundance of emitted X-rays versus their energy, the MnxGaI-xN thin films EDS. As shown in Figs. 1 and 2, results revealed the bulk structure and the distribution of the element as well as the characterized elemental composition.

The CV results of MnxGal-xN Electrodes in different salts (KF, $\mathrm{KNO}_{3}, \mathrm{KCI}, \mathrm{KSCN}, \mathrm{KCLO}$, $\mathrm{KBr}$ and $\mathrm{Kl}$ ) are shown in Table 1 of the element as well as the characterized the elemental composition.

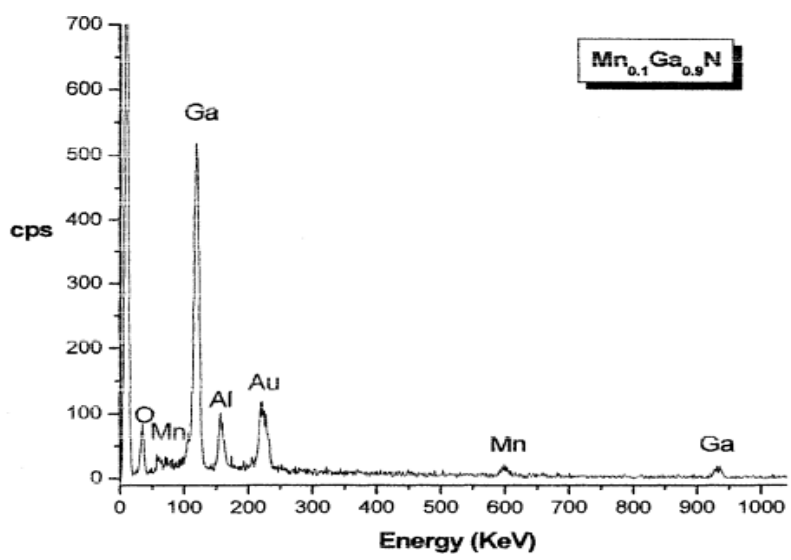

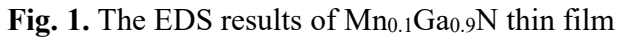

\subsection{Potentiometric response}

This section discusses the results obtained through the comparisons between the Mn0.1Ga0.9N and $\mathrm{Mn} 0.16 \mathrm{Ga} 0.84 \mathrm{~N}$ electrodes. These two electrodes were made from the same semiconductor material with different concentration of Mn incorporation. Table 2 contains the OCP values measured with both sample electrodes in various electrolyte solutions. The differences had a wide range in measured OCP results between the two MnxGal_xN sample electrodes in the selected 
electrolyte solution. This may be attributed to the heterogeneity of the MnGaN thin film across the wafer and also the series measurements were processed in the half-year period. Therefore, the performances of the two electrodes were very different. The factors that influenced the response of the electrodes were investigated in more detail in the following sections.

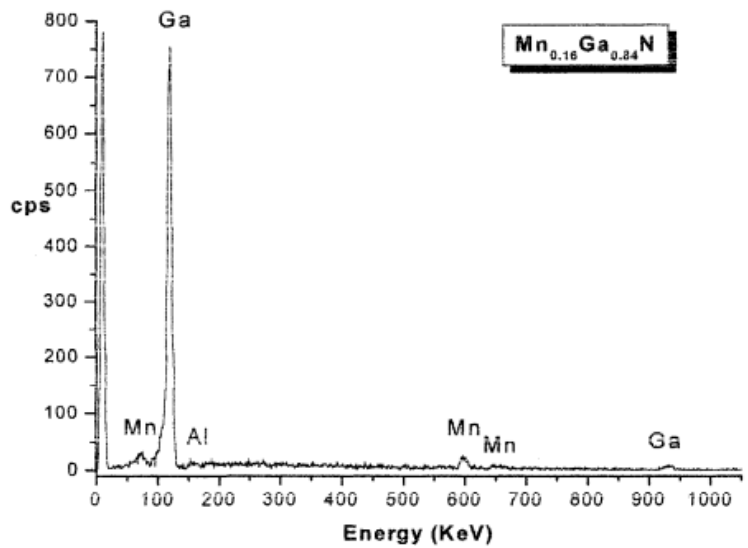

Fig. 2. EDS results of $\mathrm{Mn}_{0.16} \mathrm{Ga}_{0.84} \mathrm{~N}$ thin film

Table 1. The CV results of MnxGal-xN electrodes

\begin{tabular}{|c|c|c|c|c|c|c|c|c|}
\hline \multirow{3}{*}{ Salt } & \multicolumn{4}{|c|}{ MnD.lGauN sample electrode, SI } & \multicolumn{4}{|c|}{ MnO.16G80.84N sample electrode, 82} \\
\hline & \multicolumn{2}{|c|}{$\begin{array}{l}\text { Potential } \\
\text { Region (V) }\end{array}$} & \multicolumn{2}{|c|}{$\begin{array}{l}\text { Maximum Current } \\
\text { (fLA) }\end{array}$} & \multicolumn{2}{|c|}{$\begin{array}{l}\text { Potential } \\
\text { Region (V) }\end{array}$} & \multicolumn{2}{|c|}{$\begin{array}{l}\text { Maximum Current } \\
\text { (fLA) }\end{array}$} \\
\hline & From & To & Catbmm & Anodic & From & To & Catb1)dic & Anodic \\
\hline $\mathrm{KF}$ & -0.75 & -0.3 & -5 & 7 & - & - & - & - \\
\hline $\mathrm{KNO}_{3}$ & -0.75 & -0.3 & -1.1 & 1 & - & - & - & - \\
\hline $\mathrm{KCI}$ & -0.75 & -0.3 & -17 & 15 & -0.75 & -0.3 & -16 & 11 \\
\hline $\mathrm{HOC}_{6} 1: 4 \mathrm{COONa}$ & -0.75 & -0.3 & -0.85 & 0.5 & -0.75 & -0.3 & -10 & 4.8 \\
\hline KSCN & -0.75 & -0.3 & -0.8 & 1.25 & - & - & - & - \\
\hline $\mathrm{CH}_{3} \mathrm{COOK}$ & -0.75 & -0.3 & -0.72 & 0.9 & - & - & - & - \\
\hline KCLO. & -0.75 & -0.3 & -0.9 & 0.76 & -0.75 & -0.3 & -11 & 9 \\
\hline $\mathrm{KBr}$ & -0.75 & -0.3 & -0.85 & 0.8 & -0.75 & -0.3 & -17 & 13 \\
\hline $\mathrm{Kl}$ & -0.75 & -0.3 & -0.85 & 1.2 & -0.75 & -0.3 & -15 & 15 \\
\hline
\end{tabular}

Epitaxial layers of MnxGal_xN with concentrations of $(x=0.1$ or 0.16$)$ had been grown on $\mathrm{GaN}(0001) /$ sapphire(0001) by metalorganic chemical vapor deposition (MOCVD). X-ray Diffraction (XRD), Secondary Electron Emission/Energy Dispersive X-ray Spectrometer (SEMIEDXS), Ultraviolet (UV) measurements, and Open Circuit Potential (OCP) were analyzed using two different samples, MO0.1 Ga0.9N and MnO.16Gao.84N-. All the experiments and observations were under room temperature .

Crystalline quality and surface structure of MnxGaJ_xN were measured by XRD. The results displayed no significant deterioration in crystal quality and the increasing of surface roughness with the incorporation of $\mathrm{Mn}$. The single crystal structure and pattern distance were proved by the calculation.

The SEMIEDS results provided the interface pattern and the element distribution analysis. It was found that the Mn atoms were mostly concentrated in one section of the surface .

The energy band gaps of the samples were determined by UV measurements. It was found that the energy band gap changed in the range of $1.0 \mathrm{eV}-3.5 \mathrm{eV}$ regarding the concentration of $\mathrm{Mn}$. The Linear Combinations of Atomic Orbitals (LCAO) electron band structure of MnxGaJ_xN semiconductor compound alloy was determined. It was found that the total electron spin of the $\mathrm{Mn}$ atom was dependent on the position of the Fermi level and interband transitions in the electron 
band diagram of MnxGal_xN. The optical properties of the wurtzite MnxGal_xN were determined as well.

Table 2. Variation of OCPs with Solutions for MnxGal xN Electrodes

\begin{tabular}{|c|c|c|c|c|c|}
\hline & \multirow{3}{*}{ Cone. M } & \multirow{2}{*}{\multicolumn{2}{|c|}{$\frac{\text { Measured OCPotential }(\mathrm{mV})}{\text { SCE }}$}} & \multirow{3}{*}{$\begin{array}{l}\text { Absolute variation } \\
\text { between SI and S2 }\end{array}$} & \multirow{3}{*}{$\begin{array}{l}\text { Relative variation } \\
\text { between SI and S2 }\end{array}$} \\
\hline & & & & & \\
\hline & & SI & S2 & & \\
\hline \multirow{6}{*}{$\mathrm{KCI}$} & $10^{-6}$ & -411.78 & -316.85 & 94.93 & 30 \\
\hline & $10^{-5}$ & -458.43 & -310.03 & 148.40 & 48 \\
\hline & $10^{-4}$ & -492.18 & -373.94 & 118.24 & 32 \\
\hline & $10^{-3}$ & -515.59 & -414.21 & 101.38 & 24 \\
\hline & $10^{-2}$ & -634.78 & -445.44 & 189.34 & 43 \\
\hline & $10^{-1}$ & -662.23 & -459.20 & 203.03 & 44 \\
\hline \multirow{6}{*}{$\mathrm{HOC}_{6} \mathrm{R} . \mathrm{COONa}$} & $10^{-6}$ & -373.55 & -218.35 & 155.20 & 71 \\
\hline & $10^{-5}$ & -396.44 & -255.83 & 140.61 & 55 \\
\hline & $10^{-4}$ & -373.55 & -307.05 & 66.50 & 22 \\
\hline & $10^{-3}$ & -454.47 & -371.37 & 83.10 & 22 \\
\hline & $10^{-2}$ & -475.81 & -410.20 & 65.61 & 16 \\
\hline & $10^{-1}$ & -466.43 & -431.43 & 35.00 & 8 \\
\hline \multirow{6}{*}{$\mathrm{KCIO}_{4}$} & $10^{-6}$ & -328.30 & -314.15 & 14.15 & 5 \\
\hline & $10^{-5}$ & -398.80 & $\cdot 321.03$ & 77.77 & 24 \\
\hline & $10^{-4}$ & -481.90 & -359.93 & 121.97 & 34 \\
\hline & $10^{-3}$ & -497.70 & -436.95 & 60.75 & 14 \\
\hline & $10^{-2}$ & -526.60 & -472.87 & 53.73 & 11 \\
\hline & $10^{-1}$ & -538.00 & -494.14 & 43.86 & 9 \\
\hline \multirow{6}{*}{$\mathrm{KBr}$} & $10^{-6}$ & -364.01 & -375.22 & 11.21 & 3 \\
\hline & $10^{-5}$ & -429.28 & -309.17 & 120.11 & 39 \\
\hline & $10^{-4}$ & -486.84 & -372.83 & 114.01 & 31 \\
\hline & $10^{-3}$ & -521.85 & -428.92 & 92.93 & 22 \\
\hline & $10^{-2}$ & -556.62 & -458.06 & 98.56 & 22 \\
\hline & $10^{-1}$ & -609.23 & -469.05 & 140.18 & 30 \\
\hline \multirow{6}{*}{ KI } & $10^{-6}$ & -388.90 & -392.86 & 3.96 & 1 \\
\hline & $10^{-5}$ & -450.21 & -333.38 & 116.83 & 35 \\
\hline & $10^{-4}$ & -529.65 & -399.10 & 130.55 & 33 \\
\hline & $10^{-3}$ & -568.69 & -468.44 & 100.25 & 21 \\
\hline & $10^{-2}$ & -595.19 & -496.08 & 99.11 & 20 \\
\hline & $10^{-1}$ & -610.17 & -501.00 & 109.17 & 22 \\
\hline
\end{tabular}

OCP measurements revealed the sensitivity of the samples (MnxGat xN) on a wide range of anionic concentrations. The fabricated MnxGal_xN sample electrodes showed a good response to $\mathrm{Cl}^{\prime}, \mathrm{Br} ", \mathrm{r}, \mathrm{CI} 04-$ compared to all tested organic and inorganic anions. They also demonstrated good chemical and mechanical stability and hence, MnxGal_xN semiconductors could be employed to construct ion-selective electrodes that were both durable and easy to use. The study also showed that some of the electrode characteristics, specifically, their region of linearity and the reproducibility of OCP results, leave room for improvement and necessitate further research, as shown in Fig. 3.

In view of the above perceptions, the MnGaN surface is often thought of as a perfect transducer for the event of composite membrane-based sensors. For this, nine anion solutions (10-IM-IO-6M) were investigated via OCPs experiments of the $\mathrm{MnGaN}$ electrodes, and their responses to the Potassium, Bromine, Iodine, and perchlorate anions were also investigated under the same experimental conditions as shown in Fig. 4. The composite electrodes (sensors) had very fast and reproducible response to potassium activity changes. On the other hand, the slope of the sensor was very close to the theoretically expected one. MnGaN (0001) surface was shown to selectively interact with anions in solution, based on the potentiometric response of the MnGaN surface to 
anions with drastically different lipophilicities.

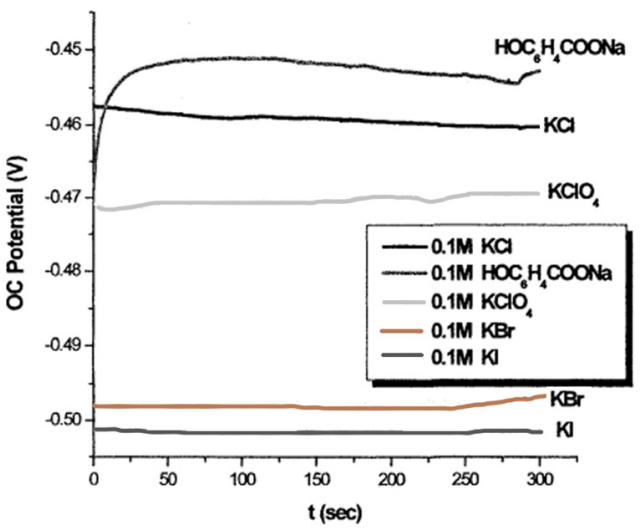

Fig. 3. The variation of OCP with time for $\mathrm{Mn}_{0.16} \mathrm{Ga}_{0.84} \mathrm{~N}$ electrode

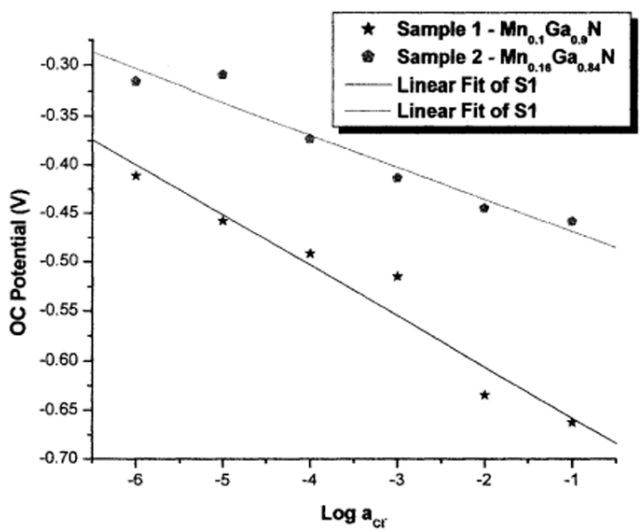

Fig. 4. Calibration curve of the $\mathrm{Mn}_{0.1} \mathrm{Ga}_{0.9} \mathrm{~N}$ and $\mathrm{Mn}_{0.16} \mathrm{Ga} 0.84 \mathrm{~N}$ in five test electrolytes

\section{Conclusions}

Chemical sensors have a wide range of applications within the control systems in the environmental and food industry. Biosensors, especially, electrochemical sensors, have become increasingly important as biochemical and biological applications always emerge. These kinds of applications include: food monitoring, automotive and medical diagnostics, home/environmental monitoring and High Throughput Screening (HTS), biological/chemical warfare (Homeland Security), and industrial applications. Potentiometric electrodes are the one type of electrochemical sensors which require, for operating, a voltmeter resolution range between $1 \mathrm{mV}$ to 1 Volt. Potentiometric sensors normally use linear sweep voltammetry and cyclic voltammetry to determine the redox potentials of the ion adsorbed at the sensor surface. Since MnGaN electrodes yielded stable cyclic voltammetry $(\mathrm{CV})$ results as well as impedance, they have great potentials in all solid-state potentiometric sensor applications. MaGaN semiconductor material has great potential to develop the GMR sensor and potentiometric sensors of control systems.

\section{References}

[1] Pérez A., Crespo-Hernández C., Reichardt C., Cabrera C., Ramos I., Ramírez L., Meador M. Synthesis, optical characterization, and electrochemical properties of isomeric tetraphenylbenzodifurans containing electron acceptor Groups. The Journal of Physical Chemistry, Vol. 115, Issue 17, 2011, p. 4157-4168.

[2] Hoffman M., Martin S., Choi W., Bahnemann D. Environmental applications of semiconductor photocatalysis. Chemical Review, Vol. 95, 1995, p. 69-96.

[3] Pern F., Frank A. Electrochemical and optical characterization of poly(3-methylthiophene): effects of solvent, anion, and applied potential. Journal of The Electrochemical Society, Vol. 137, Issue 9, 1990, p. 2769-2777.

[4] Lécuyer Christophe From clean rooms to dirty water: labor, semiconductor firms, and the struggle over pollution and workplace hazards in silicon valley Christophe Lecuyer. Information and Culture, Vol. 52, Issue 3, 2017, https://doi.org/10.7560/IC52302.

[5] Buck R., Lindneri E. Recommendations for nomeclarture of ion-selective electrodes. Pure and Applied Chemistry, Vol. 66, Issue 12, 1994, p. 2527-2536.

[6] Jim T. The essential guide to semiconductors. 1st Edition, Revolution in Miniature: The History and Impact of Semiconductor Electronics. Cambridge University Press, 1982.

[7] Matas B. Semiconductor shipments forecast to exceed 1 trillion devices in 2018. Research Bulletin, 2018 
[8] Hourani M., Alkawaldeh A. Synergistic effects of bismuth adatoms on electrocatalytic properties of electrodeposited nanostructured platinum electrodes. International Journal of Electrochemical Science, Vol. 11, 2016, p. 3555-3566.

[9] Revesz A. G., Hughes H. L. The structural aspects of Noncrystalline $\mathrm{SiO}_{2}$ films on silicon, a review. Journal of Non-Crystalline Solids, Vol. 328, Issue 3, 2003, p. 48-63.

[10] Wolf S. A., Awschalom D. D., Buhrman R. A., Daughton J. M. Spintronics, a spin-based electronics vision for the future. Science, Vol. 294, Issue 5546, 2001, p. 1488-1495.

[11] Flamini F., Spagnolo N., Sciarrino F. Photonic quantum information processing: a review. Reports on Progress in Physics, Vol. 82, 2018, p. 016001.

[12] Hu Z. G., Weerasekara A. B., Dietz N., Perera A. G. U., Strassburg M., Kane H., Asghar A., Ferguson I. T. Infrared optical anisotropy of diluted magnetic Ga1-xMnxN/c-sapphire epilayers grown with a GaN buffer layer by metalorganic chemical vapor deposition. Physical Review B, Vol. 75, 2007, p. 205320.

[13] Smith J. R., Haider M. B., Constantin C., Al-brithen H., Yang H., Trifan E., Ingram D. Ga/N flux ratio influence on $\mathrm{Mn}$ incorporation, surface morphology, and lattice polarity during radio frequency molecular beam epitaxy of (Ga,Mn)N. Journal of Applied Physics, Vol. 93, 2003, p. 5274.

[14] Chodun R., Nowakowska-Langier K., Okrasa S., Zdunek K. The application of magnetic self-filter to optimization of AIN film growth process during the impulse plasma deposition synthesis. Materials Science - Poland, Vol. 34, Issue 1, 2016, p. 110-126.

[15] Ennen I., Kappe D., Rempel T., Glenske C., Hütten A. Giant magnetoresistance: basic concepts, microstructure, magnetic interactions, and applications. Sensors, Vol. 16, Issue 6, 2016, p. 904.

[16] Bertoluzzi L., Lopez-varo P., Antonio J., Tejada J., And Bisquer L. Charge transfer processes at the semiconductor/electrolyte interface for solar fuel production: insight from impedance spectroscopy. Journal of Materials Chemistry A, Vol. 4, Issue 8, 2016, p. 2873-2879.

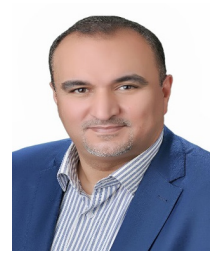

Monzer M. Krishan, Associate Professor of Mechatronics Engineering received Ph.D. degree in Informational-Measuring System/Mechatronics Engineering from National Aviation University, Kiev, Ukraine, in 2001. Now he is working at Department of Mechatronics Engineering, Faculty of Engineering Technology (FET), Al-Balqa Applied University, Amman, Jordan. Research interests: instrumentation and measurement, sensor technology, control and instrumentation, measurement and metrology, measuring devices, control, dynamics and fault diagnosis.

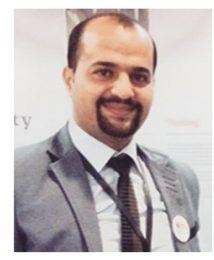

Ahmad Alkhawaldeh, Assistant Professor of chemistry received Ph.D. degree in Electroanalytical chemistry from University of Jordan, Amman, Jordan, in 2018. Now he is working at Department of chemistry, Jerash University, Jerash, Jordan. Research interests: electrochemistry catalysis, synthesis, and characterization (nanoparticle applications), development of new oxidation, and reduction system and using XRD, XPS, TGA, ICP, AAS and FTIR for detailed analysis of the organic and the inorganic residues.

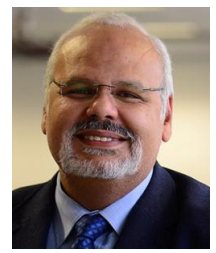

Abdel-Hamid Soliman, Associate Professor of Mechatronics Engineering. Now he is working at Department of Mechatronics Engineering, Staffordshire University, Stok on Trant, United Kingdom. Research interests: Clean Energy and Research in Environmental Studies (CERES) - TEMPUS \& water enhanced resource planning, mechatronics, smart cites. 